\title{
The multiplex nature of global financial contagions
}

\author{
R. Maria del Rio-Chanona ${ }^{1,2}$, Yevgeniya Korniyenko ${ }^{3}$, Manasa Patnam ${ }^{3}$ and Mason A. Porter ${ }^{4 *}$
}

\author{
${ }^{*}$ Correspondence: \\ mason@math.ucla.edu \\ ${ }^{4}$ Department of Mathematics, \\ University of California Los Angeles, \\ Los Angeles, CA, USA \\ Full list of author information is \\ available at the end of the article
}

\begin{abstract}
As illustrated by the 2008 global financial crisis, the financial distress of one country can trigger financial distress in other countries. We examine the problem of identifying such "systemically important" countries (i.e., countries whose financial distress can trigger further distress), which is important for assessing global financial stability. Using data on bilateral financial positions that are split by asset type, we build a multiplex global financial network in which nodes represent countries, edges encode cross-country financial assets of various types, and layers represent asset types. We examine the temporal evolution of a measure of node importance known as MultiRank centrality, and we find that several major European countries decrease in rank and that several major Asian countries increase in rank since 2008. We then develop a multiplex threshold model of financial contagions in which a shock can propagate either within a layer or between layers. We find that the number of systemically important countries can be twice as large when we take into account the heterogeneity of financial exposures (i.e., when using a multiplex network) than in a contagion on an associated aggregate global financial network (i.e., on a monolayer network), as is often examined in other studies. We also study the extent to which buffers can reduce the propagation of financial distress. Our analysis suggests that accounting for both intralayer and interlayer propagation of contagions in a multiplex structure of financial assets is important for understanding interconnected financial systems of countries.
\end{abstract}

Keywords: Financial contagions, Systemic risk, Multiplex networks

\section{Introduction}

The 2008 global financial crisis illustrated interconnectedness in the financial stability of countries. In September 2008, Lehman Brothers Holdings Inc., one of the largest investment banks in the United States, went bankrupt. By October 2008, HBOS plc and Lloyds Banking Group were bailed out in the United Kingdom (Fernando et al. 2012; Tebogo 2012). Financial distress spread to other countries, and the crisis became global (De Haas and Van Horen 2012). Financial interconnectedness continues to be pervasive. Since the middle of the 1990s, there has been a rapid expansion of cross-border financial positions (i.e., in the balances of recorded assets, liabilities, and equity), with the stocks of foreign assets and foreign liabilities increasing from about $75-77 \%$ of the world's

(c) The Author(s). 2020 Open Access This article is licensed under a Creative Commons Attribution 4.0 International License, which permits use, sharing, adaptation, distribution and reproduction in any medium or format, as long as you give appropriate credit to the original author(s) and the source, provide a link to the Creative Commons licence, and indicate if changes were made. The images or other third party material in this article are included in the article's Creative Commons licence, unless indicated otherwise in a credit line to the material. If material is not included in the article's Creative Commons licence and your intended use is not permitted by statutory regulation or exceeds the permitted use, you will need to obtain permission directly from the copyright holder. To view a copy of this licence, visit http://creativecommons.org/licenses/by/4.0/. 
gross domestic product (GDP) in 1995 to about $172-189 \%$ of the world's GDP by 2016 (Lane and Milesi-Ferretti 2017).

This significant interconnectedness and the 2008 global financial crisis both highlight the importance of identifying "systemically important" financial institutions and countries (i.e., financial institutions and countries whose financial distress can trigger distress in other institutions). In the present paper, we conduct multiplex network analysis to help improve our understanding of financial contagions and identify systemically important countries. We study both centralities and threshold models of contagions.

Many researchers have used network analysis to analyze the interconnected nature of financial systems (Markose 2012; Battiston et al. 2016; Anand et al. 2013; Wiersema et al. 2019), and several studies have used a network-based approach to estimate the systemic importance of financial entities. Perhaps most prominently, a variety of studies have developed centrality measures that are tailored to examining the importance of financial entities. For example, "impact centrality" (Kaushik and Battiston 2013) recursively takes into account that a node is more systemically important if it impacts many systemically important nodes; "default cascade impact" (Battiston et al. 2012) estimates the impact of a defaulting node; and "DebtRank" (Battiston et al. 2012) attempts to examine how "financial distress" propagates through a network, where financial distress refers to the inability or difficulty of a financial institution (or set of institutions) to pay its debt (or their debts, in the case of multiple institutions). The DebtRank of a node (or a set of nodes) gives an estimate of the monetary loss that the financial distress of a node (or set of nodes) causes due to financial interconnections. Several recent studies have used DebtRank to analyze financial networks (Li et al. 2018; Silva et al. 2018; Diem et al. 2020; Pichler et al. 2018; Poledna et al. 2018), and other studies have focused on developing different versions of DebtRank (e.g., by considering nonlinear forms of distress propagation) (Bardoscia et al. 2015, 2016).

Other studies of financial networks have used threshold models to study interconnected financial systems (Gai and Kapadia 2010; Battiston et al. 2012). Threshold models are a popular type of spreading process (Porter and Gleeson 2016) that can exhibit cascading behavior and have played an important role in the study of financial contagions (Caccioli et al. 2018). In a threshold model, each node is in one of several discrete states (in practice, two states is the most common choice), and node states update according to some rule, such as a deterministic one that is based on the states of a node's neighbors (Gleeson 2013). Typically, when modeling a financial contagion with a threshold model, nodes represent financial entities, directed edges encode lending-borrowing relationships, and the states of the nodes correspond to a solvent or defaulted status (Caccioli et al. 2018). A node updates its state according to a threshold rule: when a node loses more assets than it has equity, the node changes its state from solvent to defaulted. One reason that threshold models have been adopted widely for modeling financial contagions is that, in large networks (specifically, when the number $N$ of nodes becomes infinite), a vanishingly small fraction of initially defaulted financial institutions can cause the default of a significant fraction (i.e., a fraction that is nonzero in the $N \rightarrow \infty$ limit) of the nodes in a network (Gai and Kapadia 2010) in a "cascade"1. This idea is useful for modeling "systemic risk", which describes the risk that a significant fraction of a financial system 
defaults as a consequence of the failure of one (or a small group) of financial institutions (Poledna et al. 2015).

Notions of systemic risk have also been developed in models of interconnected financial institutions that take the form of coupled stochastic differential equations (Eisenberg and Noe 2019; Feinstein and Sojmark 2019). Other insightful approaches for studying financial contagions on networks include using branching processes to examine financial contagions from common asset holdings (i.e., overlapping portfolios) (Caccioli et al. 2014) and drawing from ideas in network ecology and disease spread to explore complexity and stability in financial networks (Haldane and May 2011).

Studies like the ones above have provided interesting insights into the dynamics of financial contagions (Caccioli et al. 2018), but most of these investigations have examined monolayer networks (i.e., ordinary networks), such as by considering lending and borrowing of a single type of asset or an aggregate of several types of assets. Multilayer networks can model interconnected structures more effectively than monolayer networks (Kivelä et al. 2014; Aleta and Moreno 2019), and they can also have significant effects on dynamical processes on them (De Domenico et al. 2016). Therefore, it is important to generalize threshold models and network measures of systemic importance (such as DebtRank) to multilayer networks. Multilayer networks include layers that can encode different subsystems and/or different types of interactions between nodes. One type of multilayer network is a multiplex network, in which interlayer edges can occur only between nodelayers (i.e., an instantiation of a node on a specific layer) that correspond to the same entity (Kivelä et al. 2014; Bianconi 2018). Several studies have used multiplex networks to study financial contagions (Aoki et al. 2010; Poledna et al. 2015; Brummitt and Kobayashi 2015; Bargigli et al. 2015; Greenwood et al. 2015). Existing research on multiplex financial networks has studied microlevel and country-specific data (e.g., analyzing a network of banks at a national level) (Diem et al. 2020; Poledna et al. 2015), examined DebtRank for different layers of a multiplex network (Poledna et al. 2015), and applied threshold models but with a focus on unweighted and overly simplistic synthetic networks (e.g., by using layers that consist of Erdős-Rényi networks) (Brummitt et al. 2012; Brummitt and Kobayashi 2015).

Most studies of threshold models on financial networks have focused on monolayer networks (Gai and Kapadia 2010; Battiston et al. 2012), although there have been some extensions of threshold models to multiplex networks. For example, Brummitt et al. (2012) examined threshold dynamics on unweighted multiplex networks in which a node activates if, in any layer, its fraction of active neighbors exceeds a given threshold. Lee et al. (2014) extended this model by including some nodes that activate only if their fraction of active neighbors in each layer is larger than a specified threshold. Yağan and Gligor (2012) examined a multiplex threshold model with a single update rule that incorporates all edge types. In applications to financial networks, Brummitt and Kobayashi (2015) considered a multiplex threshold model with two layers in which different layers correspond to different debt seniorities (i.e., to the chronological order of debt repayments when there are sales or defaults). 
As in Brummitt et al. (2012), we examine a multiplex threshold model in which a contagion can spread almost immediately across layers ${ }^{2}$. In our model, the default of node-layer $(i, \alpha)$ at time step $t$ can cause node-layers $(i, \beta)$ with $\beta \neq \alpha$ to default in the next time step.

The studies by Brummitt et al. (2012), Yağan and Gligor (2012), Lee et al. (2014), and Brummitt and Kobayashi (2015) illustrated that multilayer financial networks can be more vulnerable than monolayer networks to large-scale cascades in some dynamical processes. Additionally, using empirical data, Poledna et al. (2015) and Leon et al. (2014) reported that systemic risk in the Mexican and Columbian banking systems, respectively, are underestimated significantly when one examines monolayer networks that encode only one type of financial asset, rather than studying a multiplex network that encodes multiple types of financial assets.

In the present paper, we use multilayer network analysis to examine how financial distress propagates between countries. In particular, we simulate financial shocks and their propagation through a network. We start with a single financially distressed country and examine the ensuing distress of other countries. Our primary aim is to examine the socalled "global financial system" - an interconnected network of lending and borrowing of financial assets between countries - and perform multilayer network analysis to identify systemically important countries. We study multiplex networks, so interlayer edges can occur only between node-layers that correspond to the same country. To examine important countries, we employ the MultiRank centrality measure (Rahmede et al. 2018), and we extend multilayer threshold models (Brummitt et al. 2012; Brummitt and Kobayashi 2015; Korniyenko et al. 2018) for the propagation of financial contagions by incorporating a mechanism for shock propagation between different layers.

We go further than previous research by generalizing studies of multiplex threshold models that examined almost immediate contagion spreading between layers (Korniyenko et al. 2018; Brummitt and Kobayashi 2015). In our generalization, we include a threshold rule for the interlayer spreading of a contagion. When this threshold is 0 , our model reduces to a situation with almost immediate spreading of contagions between layers. Another novelty of our model is that we incorporate a threshold rule for intralayer contagions that depends on the "capital adequacy" of a country's financial system; this gives a rough measure of a country's ability to withstand shocks. This is important, as recent work (Farmer et al. 2020) has illustrated that countries with adequate capital buffers can use them to absorb shocks and reduce systemic risk during financial distress.

By considering different layers and allowing spreading between them, we are able to examine financial contagions that take the heterogeneity of financial exposures into consideration. Specifically, we study contagion dynamics on a multiplex network whose layers (which each represent one type of asset) encode cross-country assets in equity, debt, and banks' loans and deposits.

Our paper proceeds as follows. We start by giving a brief overview of multilayer and multiplex networks. We then explain how we build a multiplex network in which nodes are countries, edges encode asset holdings between countries, and layers represent asset types. As in Korniyenko et al. (2018), we build multiplex and monolayer networks

${ }^{2}$ For additional details, see the present paper's associated technical report (Korniyenko et al. 2018). That report also includes a descriptive analysis of the global financial system (which is an interconnected network of lending and borrowing of financial assets between countries). 
using data from the International Monetary Fund (IMF) and the Bank for International Settlements (BIS) ${ }^{3}$.

We also explain MultiRank (Rahmede et al. 2018), an algorithm for identifying important nodes in a multilayer network that we use for an initial exploratory analysis. We then develop and study a multilayer threshold model. We then discuss our results from the MultiRank algorithm and our threshold model, and we complete our paper with a short concluding discussion and an appendix with additional details.

\section{Multiplex networks}

Multilayer networks are an extension of ordinary (i.e., monolayer) networks that allow one to encode multiple types of edges, multiple subsystems, and other complications (Kivelä et al. 2014; Aleta and Moreno 2019; Porter 2018). A monolayer network $G=(\mathcal{V}, E)$ consists of a set $\mathcal{V}$ of nodes and a set $E \subseteq \mathcal{V} \times \mathcal{V}$ of edges that connect the nodes to each other. In contrast to monolayer networks, multilayer networks include "layers" (which we use to encode different types of edges), node-layer tuples (which we call "node-layers" as a shorthand) $(i, \alpha)$ that represent node $i$ on layer $\alpha$, and both intralayer and interlayer edges. Intralayer edges connect node-layer tuples within a layer, whereas interlayer edges connect node-layer tuples from different layers. In a directed multilayer network, one writes an edge as an ordered pair of node-layers. Following the convention of Kivelä et al. (2014), we use Latin letters to denote nodes and Greek letters to denote layers.

Multiplex networks are node-aligned multilayer networks, as interlayer edges can only connect node-layer tuples of the same node index (i.e., ones that correspond to the same entity). In a multiplex network, each layer represents a particular interaction type and can itself be viewed as a monolayer network. In the present study, we work with multiplex networks without interlayer edges, so we are using edge-colored multigraphs. We encode the information of such a multiplex network by a set $\mathcal{A}=\left\{A^{[1]}, \ldots, A^{[L]}\right\}$ of adjacency matrices, where $L$ denotes the number of layers.

\section{A multiplex global financial network}

We construct a multiplex network of financial assets between countries. In this "global financial multiplex network", each node represents a country and each layer represents an asset type. An edge from node $i$ to node $j$ in layer $\alpha$ indicates that country $i$ has an asset of type $\alpha$ in country $j$. The edges are directed, and we weight them by their asset value (in United States dollars [USD]). To construct a multiplex global financial network (one for each year from 2008 to 2018), we use data from the IMF and BIS. Specifically, we use annual data from the IMF Coordinated Direct Investment Survey (CDIS) (International Monetary Fund 2018a) and the IMF Coordinated Portfolio Investment Survey (CPIS) (International Monetary Fund 2018b) on countries' cross-border equity and debt holdings. We use quarterly data from the BIS International Banking Statistics on countries' cross-border bank loans and deposits (Bank for International Settlements 2018).

Using these data sets, for each year, we construct a directed and weighted multiplex network of three layers and 131 countries. The layers correspond to different types of

\footnotetext{
${ }^{3}$ Our multiplex network has three layers. We use a single equity layer, which encodes both cross-border direct and cross-border portfolio investments in equity. Similarly, we construct the debt layer from both cross-border direct and cross-border portfolio investments in debt assets. In Korniyenko et al. (2018), our multiplex network had five layers, as the equity layer and debt layer were each split into two layers.
} 
financial assets: (i) cross-border portfolio and direct investments in equity; (ii) crossborder portfolio and direct investments in debt assets; and (iii) cross-border bank loans and deposits. Our use of different types of financial assets is motivated by empirical research. Prior research has established that different types of financial assets have different volatilities, so the loss of some types of asset can cause more financial distress than the loss of other types (Becker et al. 2009; Claessens et al. 1995). A country's response to financial distress depends on the type of financial assets that are impacted. For example, in response to distress in banking assets, it may be sufficient to direct a policy action exclusively at banks. However, a country would typically respond differently to distress in other asset types. For example, such a response may include a fiscal package that compensates for distress by providing government transfers, credit, or tax reliefs to support the economy.

We also use data from the IMF's Financial Soundness Indicators (FSI) database (International Monetary Fund 2018c) to obtain capital adequacy ratios (CARs) of the financial system. The CARs for some countries are missing in the FSI database, so we retain only countries where this data is available for our network analysis. This yields the 131 countries in our networks. See Fig. 1 for a schematic representation of our multiplex global financial network. We encode the information in a multiplex network with layer adjacency matrices

$$
A_{i j}^{[\alpha]}= \begin{cases}\omega_{i j}^{[\alpha]}, & \text { if country } i \text { holds assets of type } \alpha \text { in country } j \\ 0, & \text { otherwise }\end{cases}
$$

where $\omega_{i j}^{[\alpha]}$ is the value in USD of the assets of type $\alpha$ that country $i$ has invested in country $j$. These multiplex networks do not have interlayer edges, so they are edge-colored multigraphs.

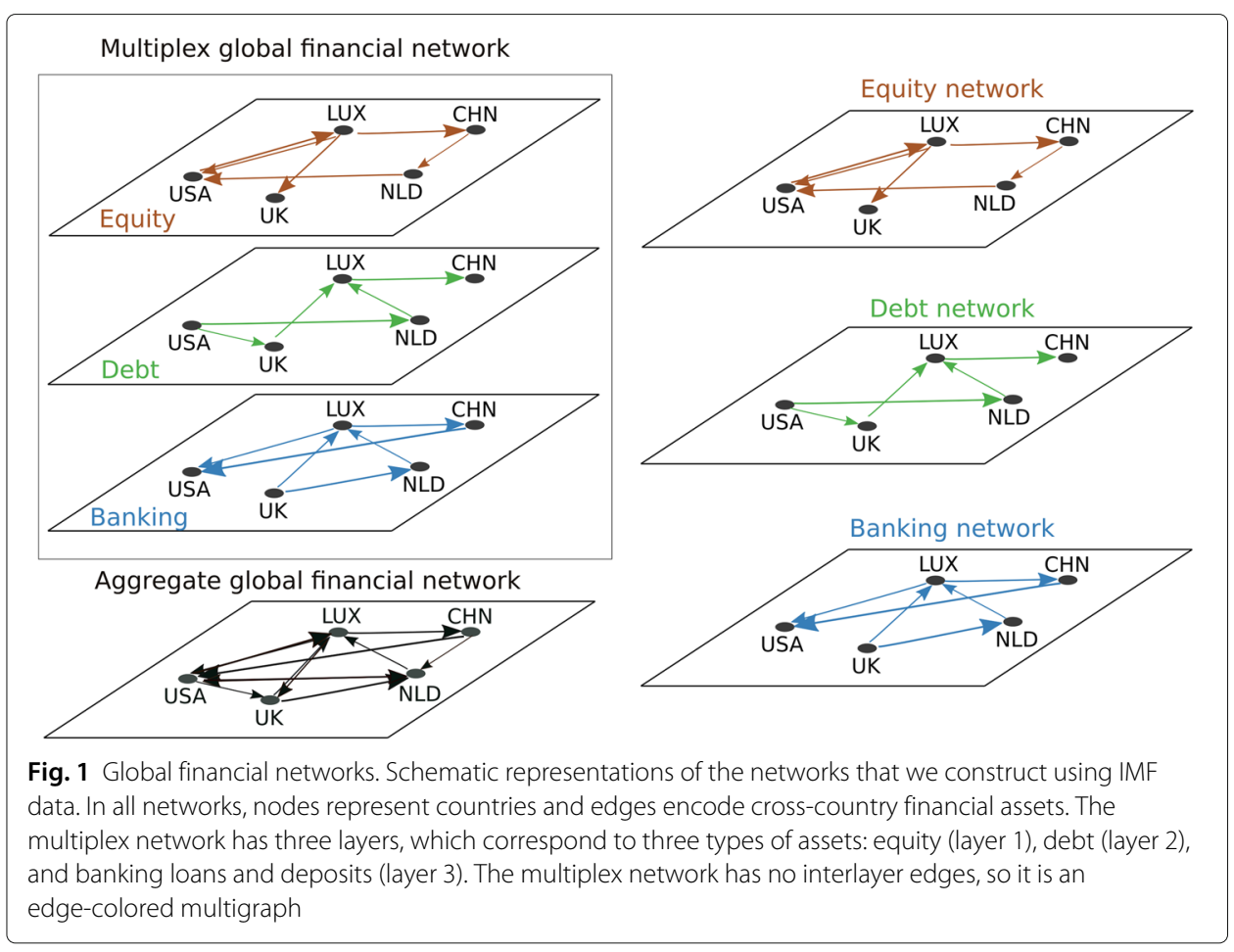


Given a multiplex global financial network, one can compute important financial quantities. For example, the value of the assets of type $\alpha$ that country $i$ holds in other countries is

$$
a_{i \alpha}=\sum_{j} A_{i j}^{[\alpha]},
$$

and the total value of the external assets that country $i$ holds is

$$
a_{i}=\sum_{\alpha} a_{i \alpha} .
$$

In addition to a multiplex global financial network, we construct associated monolayer networks by aggregating the layers of our multiplex global financial network to obtain an adjacency matrix as the sum of the adjacency matrices of the three layers. In other words, we aggregate all assets equally without distinction. This yields a single adjacency matrix A with elements $A_{i j}$, which encode the assets in USD that country $i$ has invested in country $j$ for all countries $i$ and $j$. We refer to this network as the "aggregate global financial network". We also examine each layer as a separate monolayer network. We refer to these monolayer networks from layers 1, 2, and 3 as the "equity", "debt", and "banking" networks, respectively.

\section{Identifying systemically important countries}

In this section, we discuss our methods for identifying systemically important countries, which can cause a significant fraction of the countries to become financially distressed. As an initial approach, we use MultiRank (Rahmede et al. 2018), a centrality measure for multiplex networks. We then study a multiplex threshold model, which allows a contagion to spread between different layers.

\section{MultiRank}

Centrality diagnostics measure the relative importances of nodes (or edges or other structures) in a network (Newman 2018), and it is thus natural to use centrality measures to try to identify systemically important nodes. To identify important countries in a monolayer network, we calculate PageRank centrality, a measure of a node's importance that is based on a random walk with teleportation (Newman 2018; Gleich 2015). In our calculations, we use the teleportation-parameter value $\kappa=0.15$, where $1-\kappa$ is the probability that a random walker moves to an adjacent node and $\kappa=0.15$ is the probability that a random walker teleports to any node (which we choose uniformly at random) in the network (Gleich 2015). The choice of $\kappa=0.15$ is the most common choice in PageRank, although it is an arbitrary value.

To examine important countries and important asset types in our multiplex global financial network, we calculate MultiRank (Rahmede et al. 2018), a centrality measure that was inspired by PageRank, to rank both nodes and layers. One assigns a MultiRank centrality to each node $i$ as a whole, rather than to each node-layer $(i, \alpha)$.

Several multilayer centrality measures have been developed (Kivelä et al. 2014; Bravo and Óskarsdóttir 2020). We use MultiRank both because it is easy to implement and because it allows us to rank both nodes and layers.

We briefly explain how to compute node $i$ 's MultiRank $x_{i}$ and layer $\alpha$ 's MultiRank $z^{[\alpha]}$. MultiRank assigns a large centrality to a node when central nodes point to it in layers with 
high influence (Rahmede et al. 2018). One can determine the MultiRank (i.e., "influence") of a layer in one of two ways, depending on a parameter $\delta \in\{-1,1\}$. If $\delta=1$, layers tend to be more influential if very central nodes in it are adjacent to each other. By contrast, if $\delta=-1$, layers tend to be more influential if there are few nodes with nonzero in-degree and if the nodes that do have in-edges have large centralities. In the present paper, we use $\delta=1$, because we consider a type of asset to be more important when important countries have an exposure to it.

For multiplex networks in which each node has an out-degree of at least 1 in at least one layer, we compute both node MultiRank and layer MultiRank by solving the following set of equations:

$$
\begin{aligned}
z^{[\alpha]} & =\frac{1}{N}\left(W^{[\alpha]}\right)^{\phi}\left(\sum_{i=1}^{n} B_{\alpha i}^{\mathrm{in}}\left(x_{i}\right)^{\delta \gamma}\right)^{\delta}, \\
x_{i} & =\kappa \sum_{j=1}^{n} \frac{G_{j i}}{k_{j}} x_{j}+\frac{1-\kappa}{n},
\end{aligned}
$$

where $z^{[\alpha]}$ is layer $\alpha$ 's MultiRank, $N$ is a normalizing factor, $W^{[\alpha]}=\sum_{i, j} A_{i j}^{[\alpha]}$, the adjacency matrix is $G=\sum_{\alpha=1}^{L} z^{[\alpha]} A_{i j}^{[\alpha]}$, there are $n$ nodes, the out-degree of node $j$ is $k_{j}=\sum_{i} G_{j i}$, and the normalized in-strength $B_{\alpha i}^{\text {in }}$ of node $i$ in layer $\alpha$ is

$$
B_{\alpha i}^{\mathrm{in}}=\frac{\sum_{j} A_{j i}^{[\alpha]}}{W^{[\alpha]}} .
$$

As in the PageRank algorithm, $\kappa$ denotes the probability that a random walker moves to an adjacent node and $1-\kappa=0.15$ is the teleportation probability, but now we use the adjacency matrix $G_{i j}$. The parameter $\gamma>0$ influences the contributions of smallcentrality nodes by either enhancing $(\gamma>1)$ or suppressing $(\gamma<1)$ them, and $\phi \in$ $\{0,1\}$ determines whether one considers (if $\phi=1$ ) or does not consider (if $\phi=0$ ) the total edge weight in layer $\alpha$ (Rahmede et al. 2018). In the present paper, we suppose that edge weights are important (because they encode financial exposures), so we use $\phi=1$. For simplicity, we choose $\gamma=1$, which implies that nodes contribute to layer influence (i.e., layer MultiRank) in a linear fashion. For details about how to derive this form of the MultiRank algorithm, see the "MultiRank" section of the appendix.

MultiRank has been used to study various multiplex networks (Bonaccorsi et al. 2019; van Lidth de Jeude 2019), and it is useful for exploring important countries in a multiplex financial network. However, to properly study a global financial contagion, it is important to use tools that are designed specifically for that topic.

\section{Systemic importance of nodes}

Several centrality measures have been developed to study the systemic importance of financial entities in networks (Battiston et al. 2012; Bardoscia et al. 2015; Bardoscia et al. 2016; Battiston et al. 2012). Most notably, DebtRank, a scalar quantity that one can compute either for a single node or for a set of nodes, gives an estimate of the monetary loss from the financial distress of that node (or those nodes).

Unfortunately, DebtRank has an issue that makes it inadequate for our problem ${ }^{4}$. DebtRank is not additive, in the sense that the DebtRank of the set $\{i, j\}$ of two nodes ( $i$ and

${ }^{4}$ Additionally, in the context of DebtRank, shocks tend to propagate only a small distance in densely connected networks. Therefore, DebtRank also has other features that one may wish to modify when studying a problem like ours. 
j) can be smaller than the DebtRank of the individual nodes $i$ or $j$. This lack of additivity makes it difficult to generalize DebtRank to multiplex networks. In particular, a straightforward extension of DebtRank to multiplex networks can yield a larger DebtRank for node-layer $(i, \alpha)$ than for the set $\{(i, \alpha)\}_{\alpha=1}^{L}$ of node-layers. In such a generalization, the financial distress of a country in one layer causes a larger monetary loss than the loss from a financial distress of that country in all layers. From an economic perspective, this seems unreasonable. For details about this issue, see the "DebtRank" section of the appendix.

Given the above issue, we propose a different approach: we develop a threshold model of financial contagions for multiplex networks that can be informative about the systemic importance of different countries in the global financial network. We model the propagation of a financial contagion and identify the countries that cause a sufficiently large fraction of the network to become distressed. In particular, we examine the fraction of countries whose assets have a total value that exceeds $90 \%$ of the total value of all assets in a network.

A multiplex threshold model of financial contagions. We develop a discrete-time, deterministic model of threshold dynamics; and we simulate it on multiplex networks that are weighted and directed. Like the nodes in the Watts threshold model (WTM) of a social contagion (Watts 2002) and the Gai-Kapadia model of a financial contagion (Gai and Kapadia 2010), node-layers in our networks have binary states: node-layers (i.e., country-assets) can be either undistressed or distressed ${ }^{5}$. The state of node-layer $(i, \alpha)$ at time $t$ is

$$
s_{i \alpha}(t)=\left\{\begin{array}{l}
0, \text { if }(i, \alpha) \text { is undistressed at time } t \\
1, \text { if }(i, \alpha) \text { is distressed at time } t
\end{array}\right.
$$

As in the Gai-Kapadia model (Gai and Kapadia 2010), in which nodes have capital buffers, we suppose that each country has assets in each layer that it holds as capital buffers. The purpose of these buffers is to absorb some amount of loss and avoid distress. Inspired by how national regulators track the CARs of banks based on information about their risky assets, we suppose that the value of the capital buffers that are held by country $i$ in layer $\alpha$ is proportional to the value $a_{i \alpha}$ of the assets that it holds in that layer.

We model the spread of a contagion as follows. If a country loses more assets in a layer than its layer buffer, the country becomes distressed and loses all assets. Additionally, if the sum of the lost assets of a country over all layers exceeds a fraction $c_{i}$ of its total assets $a_{i}$, then the country becomes distressed in all layers. This last contagion mechanism is based on risk propagation within countries: when investors perceive that the risk of financial distress of a country increases, the value of that country's assets decreases.

With these ideas in mind, our spreading rule for a financial contagion is

$$
s_{i \alpha}(t+1)=\left\{\begin{array}{l}
1, \text { if } \frac{\operatorname{loss}_{i \alpha}(t)}{a_{i \alpha}}>b_{i \alpha} \\
1, \text { if } \frac{\operatorname{loss}_{i}(t)}{a_{i}}>c_{i} \\
0, \text { otherwise }
\end{array}\right.
$$

where $s_{i \alpha}(t)$ denotes the state of country $i$ in layer $\alpha$ at time $t$, the quantity $b_{i \alpha}$ is the fraction of assets that are held by country $i$ in layer $\alpha$ as cash reserves,

${ }^{5}$ Several studies of financial networks of banks and firms that used threshold models have employed the terms "solvent" and "defaulted" instead of "undistressed" and "distressed" (Lee et al. 2014; Brummitt and Kobayashi 2015; Gai and Kapadia 2010). However, because we are studying financial networks of countries, rather than banks or firms, we prefer to use the terms "undistressed" and "distressed". 


$$
\operatorname{loss}_{i \alpha}(t)=\sum_{j} A_{i j}^{[\alpha]} s_{j \alpha}(t)
$$

is the monetary value of the lost assets by the end of time $t$ (i.e., including both at and before time $t$ ) for country $i$ in layer $\alpha$, and

$$
\operatorname{loss}_{i}(t)=\sum_{\beta} \operatorname{loss}_{i \beta}(t)
$$

is the monetary value of the lost assets of country $i$ by the end of time $t$.

We refer to each $b_{i \alpha}$ (which encode capital buffers) as a "horizontal threshold" and each $c_{i}$ (which encode intra-country risk-propagation buffers) as a "vertical threshold". To the best of our knowledge, our incorporation of vertical thresholds is a novel feature of our multiplex threshold model. We expect that the idea of having both vertical and horizontal thresholds will also be useful for studying other types of contagions, such as the spread of information and ideas in multichannel communication networks.

For our monolayer financial networks, we run a similar contagion model, but we now neglect the interlayer (i.e., intracountry) contagions. Specifically, we use the update rule

$$
s_{i}(t+1)=\left\{\begin{array}{l}
1, \text { if } \frac{\operatorname{loss}_{i}(t)}{a_{i}}>b_{i} \\
0, \text { otherwise }
\end{array}\right.
$$

where $s_{i}(t)$ denotes the state of country $i$ at time $t$, the quantity $a_{i}$ denotes the value of the assets of country $i$, and $b_{i}$ is a threshold that represents the capital buffer of country $i$. The total value of the financial assets that are lost by country $i$ in a monolayer network is

$$
\operatorname{loss}_{i}(t)=\sum_{j} A_{i j} s_{i}(t) .
$$

Initial conditions and parameters of the models. To calibrate the horizontal thresholds (i.e., capital buffers), we use CAR data. A CAR measures the ratio of a bank's capital to its risk-weighted assets ${ }^{6}$.

The Basel Committee on Banking Supervision (BCBS) recommends minimum CARs that financial institutions must maintain to have a sufficient buffer to provide a cushion against losses due to financial distress (Basel Committee on Banking Supervision 2019).

Although CARs are only for banks, we use them to calibrate the horizontal thresholds in the layers as a proxy for the heterogeneity in the robustness of different countries towards shocks. We assume that $b_{i \alpha}=b_{i}=\mathrm{CAR}_{i}$ for all $\alpha$ as a baseline scenario. We then consider several scalar multiples of $\mathrm{CAR}_{i}$ as the value of $b_{i}$ to examine alternative scenarios. For the vertical thresholds, we suppose that the countries are homogeneous (i.e., that $c_{i}=c$ for all $i$ ). As a reference value, we take $c_{i}=1 / L$, where $L$ is the number of layers in our multiplex global financial network. In the "Results" section, we also examine our threshold model using different values of the vertical threshold $c$.

We run our threshold model on our multiplex global financial network and on the monolayer networks (i.e., the aggregate, equity, debt, and banking networks). All of these networks are weighted and directed. We initialize our model with a single country $i^{*}$ that is distressed in all layers, and we measure the subsequent number of countries that become distressed. Our model has a deterministic update rule, so each individual

\footnotetext{
${ }^{6}$ Regulators calculate risk-weighted assets by considering a bank's assets, evaluating the risk levels of those assets, and then assigning a weight. The risk-weighted value of an asset is the value of the asset multiplied by a weight that is assigned to it by a regulator. When there are several assets, one calculates a weighted sum of those assets.
} 
simulation has a deterministic outcome. Once a node-layer is distressed (and, in each monolayer network, once a node is distressed), it remains distressed for the rest of a simulation. A simulation ends when no node-layers change their state anymore, and we use $\tau$ to denote the time step at which a simulation ends. We consider a country to be distressed when it is distressed in all layers. The extent $R\left(i^{*}\right)$ of a contagion from a seed country $i^{*}$ is the percent of assets that are collectively held by the distressed countries at the end of the simulation. That is,

$$
R\left(i^{*}\right)=100 \times \frac{\sum_{i} a_{i} \prod_{\alpha} s_{i \alpha}(\tau)}{\sum_{i} a_{i}} .
$$

We say that country $i^{*}$ is systemically important when $R\left(i^{*}\right)>90 \%$. Analogously, for the monolayer networks, the extent of a contagion is

$$
R\left(i^{*}\right)=100 \times \frac{\sum_{i} a_{i} s_{i}(\tau)}{\sum_{i} a_{i}} .
$$

\section{Results}

We examine the temporal evolution of our multiplex global financial network in two ways. We first use MultiRank to examine how layers and countries change in rank over time. For each year in our data set, we compare the MultiRank values of countries in the multiplex global financial network with the PageRank values of countries in the aggregate global financial network. We then study global contagions using our threshold model and examine the number of systemically important countries. We do this for the multiplex global financial network and for the equity, debt, banking, and aggregate global financial networks.

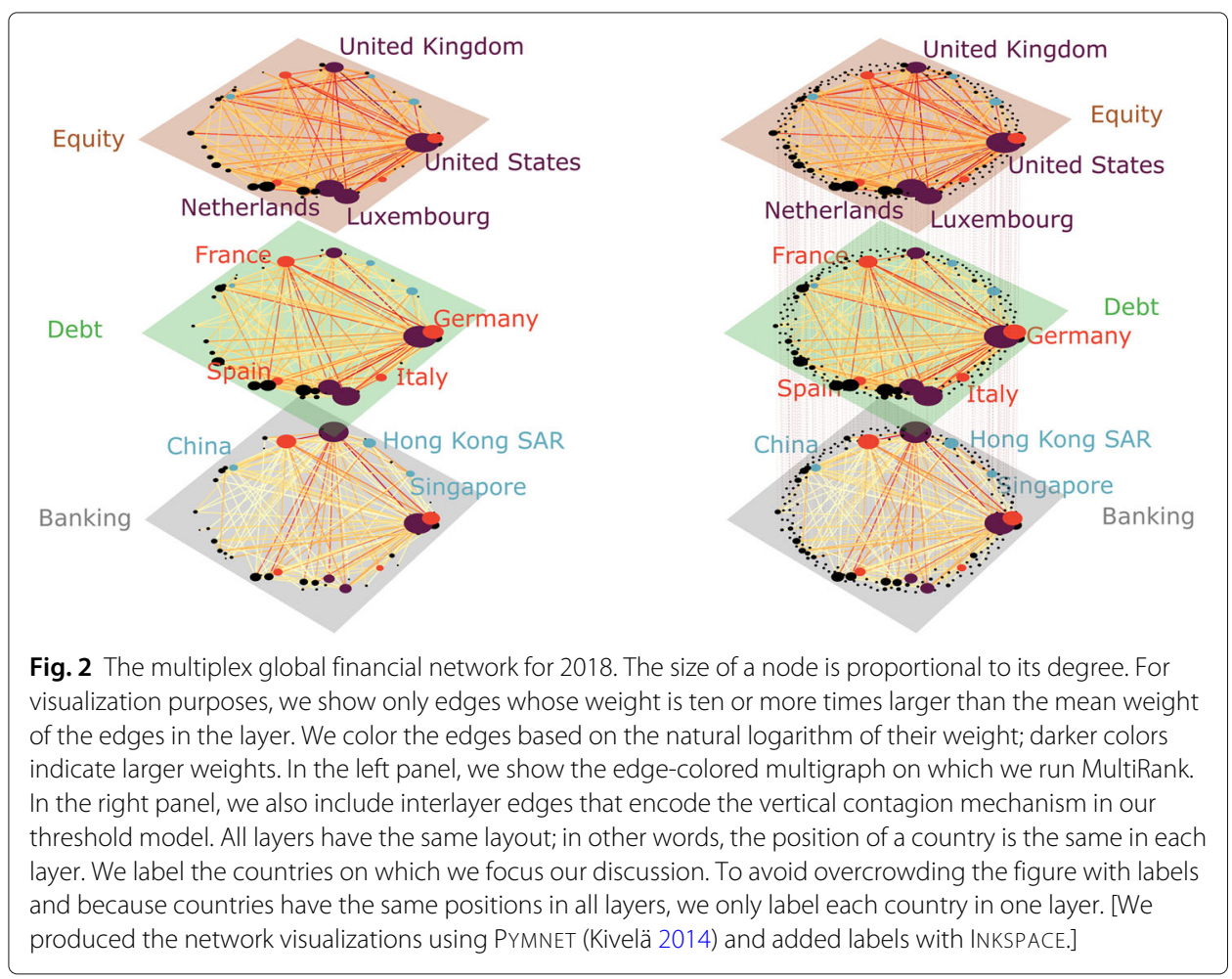




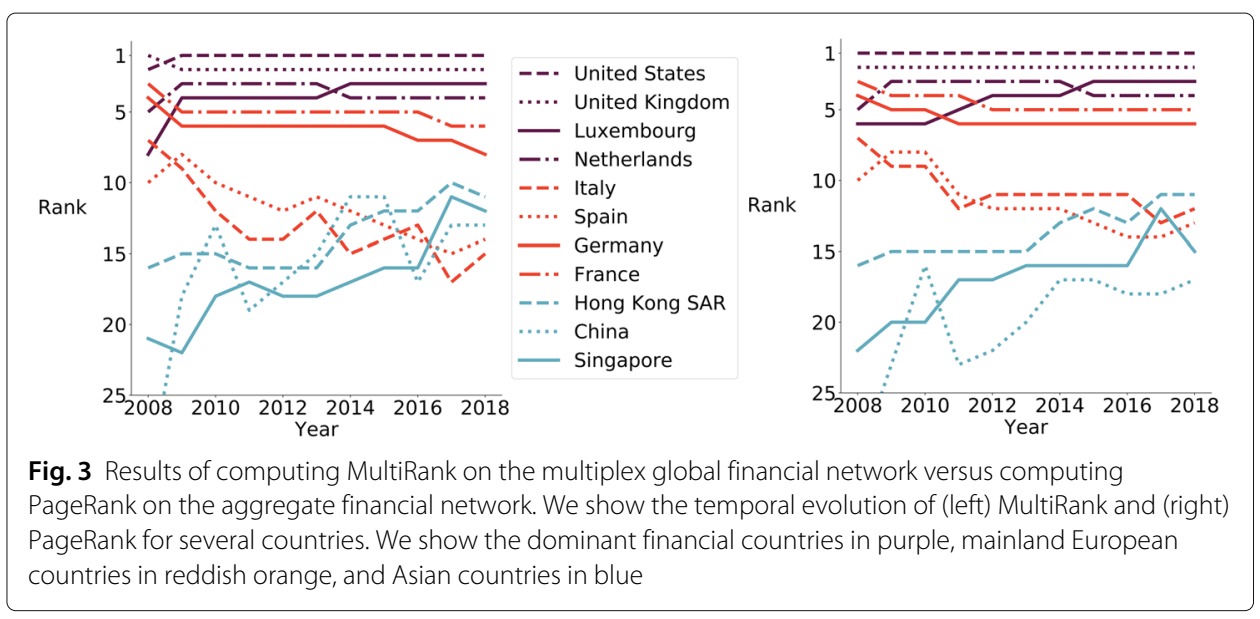

In Fig. 2, we show a visualization of the multiplex global financial network for 2018. We label and highlight the countries on which we focus our discussion. Visualizing this multiplex network illustrates the heterogeneity across layers. For example, the degree of the United Kingdom is considerably larger in the banking layer than in the equity layer. By contrast, the opposite is the case for the Netherlands and Luxembourg.

\section{MultiRank evolution}

Our centrality analysis yields different results for the multiplex financial network than it does for any of the four monolayer networks (the equity, debt, banking, and aggregate networks). For brevity, we focus on discussing the differences between the multiplex global financial network and the aggregate monolayer network. Computing MultiRank and PageRank for the multiplex and aggregate networks, respectively, produces results that correlate strongly with each other, with a Kendall rank correlation coefficient of above 0.90 for each year. (See the "Correlation between MultiRank and PageRank for the Global Financial Network" section of the appendix for details.) There is also a slight downward trend in the correlation over the years. Both centrality measures illustrate that (i) the United States, the United Kingdom, Luxembourg, and the Netherlands have the top ranks for most years; (ii) several major European countries decrease in centrality over time; and (iii) several major Asian countries increase in centrality over time. Notably, computing MultiRank for the multiplex global financial network better highlights the emerging role of Asian countries than computing PageRank for the aggregate network. In particular, according to MultiRank, Hong Kong SAR, China, and Singapore rank higher than Italy and Spain in 2018 (see Fig. 3).

To better understand the emerging role of Asian countries as systemically important countries, we examine changes over time in the different layers of the multiplex global financial network. Since the 2008 global financial crisis, which started in the United States banking sector, many countries have made an effort to deleverage banking assets, especially in Europe $e^{7}$. Another trend that we observe (see the right panel of Fig. 4) is that the fraction of the asset value in the equity layer grows over time, whereas the fractions in the other two layers decrease over time (excluding the year 2008) ${ }^{8}$. Our calculations of

\footnotetext{
${ }^{7}$ The persistent aggregate decline in cross-border banking claims of European banks was discussed in Lund et al. (2013), Forbes (2014), Forbes et al. (2017), Lane and Milesi-Ferretti (2017), and Mallaby 2016.

8 Some countries only started systematically reporting CDIS data to the IMF in 2009. This explains the missing equity data for some countries in 2008. The issue of "missing" data is visible in Fig. 4, where we observe that the equity assets have a very small total value in 2008. Consequently, we exclude 2008 from our discussion of the importances of the layers.
} 


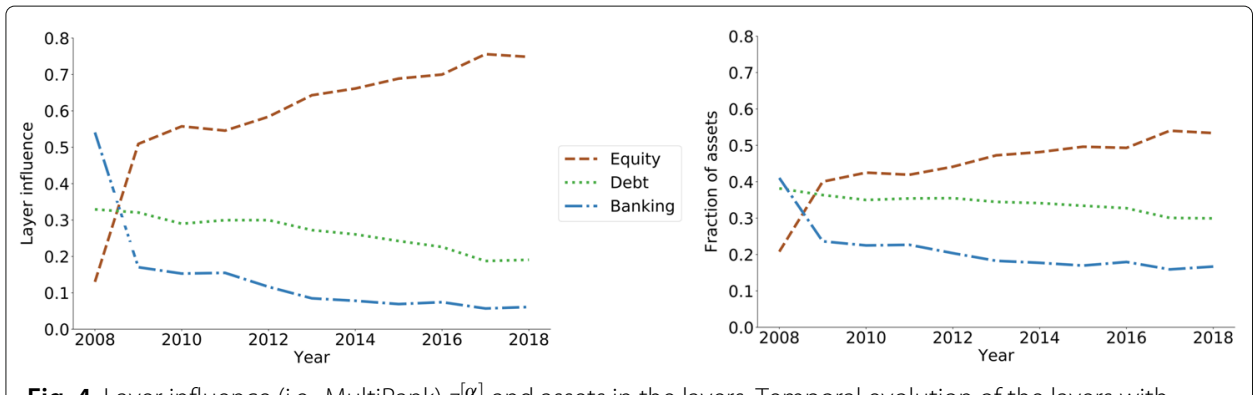

Fig. 4 Layer influence (i.e., MultiRank) $z^{[\alpha]}$ and assets in the layers. Temporal evolution of the layers with respect to (left) the influence of the layers and (right) the fraction of assets in each layer

MultiRank also illustrate an increasing centrality of the equity layer. (See the left panel of Fig. 4.) As the banking layer decreases in importance, some Asian countries (which are more central in the equity and debt layers) increase in centrality. We interpret the MultiRank and the fraction of the asset value of a layer $\alpha$ as measures of the relative importance of a layer with respect to other layers. In this context, we find that MultiRank amplifies the growth of the relative importance of the equity layer over time. Although the fraction of the asset value of the equity layer increases only from about 0.4 in 2009 to about 0.53 in 2019, the influence (i.e., MultiRank) of the equity layer increases from about 0.51 in 2009 to about 0.75 in 2019 .

\section{Threshold contagion models}

Number of systemically important countries. For each year of our data, we run our threshold models for the multiplex global financial network and for each monolayer network (namely, the equity, debt, banking, and aggregate networks). Recall that we use update rule (7) for the multiplex network and update rule (10) for each monolayer network. For the multiplex network, we consider several different values of the (homogeneous) vertical threshold. As a reference choice, we take $c=0.33 \approx 1 / 3=1 / L$, but we also consider vertical thresholds of $0.5,0.66$, and 0.80. As we show in Fig. 5 , for $c=0.33$,

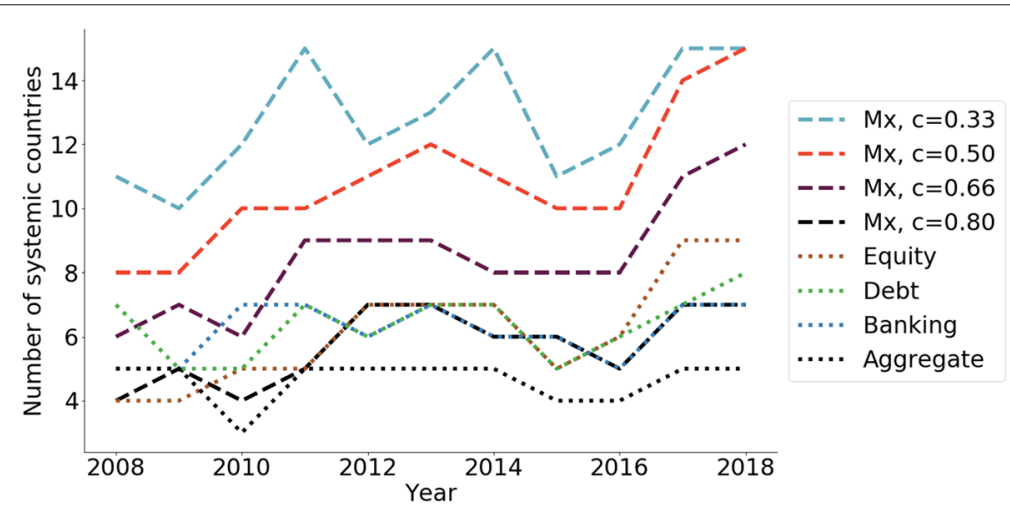

Fig. 5 The number of systemically important countries (which we abbreviate as "systemic countries" in the axis label) over time in the multiplex global financial network and in its associated monolayer networks. We show simulations on the multiplex global financial network (which we abbreviate by "Mx" in the legend) using dashed curves and simulations on the monolayer networks using dotted curves. For the multiplex network, blue, reddish orange, purple, and black curves correspond to vertical thresholds of $0.33,0.50,0.66$, and 0.80 , respectively. For the monolayer networks, brown, green, blue, and black curves correspond to the equity, debt, banking, and aggregate networks, respectively 


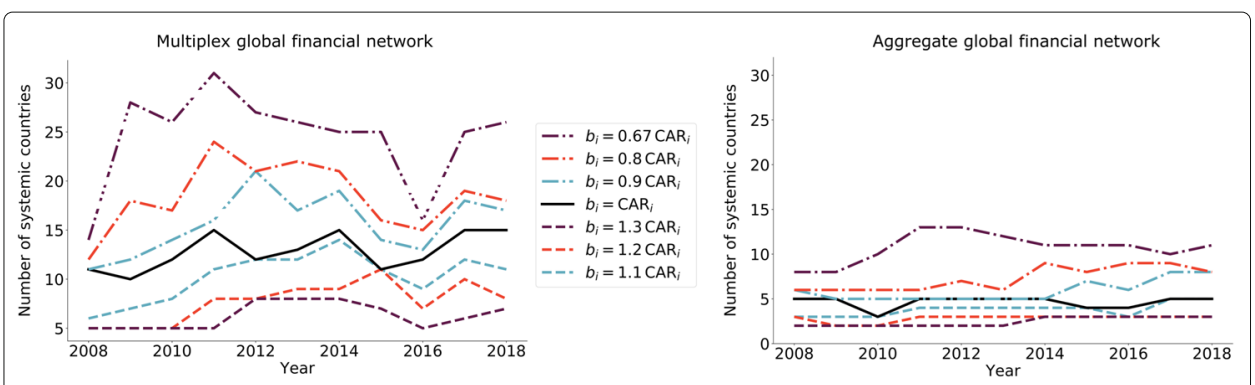

Fig. 6 Systemically important countries and horizontal thresholds. We show the number of systemically important (i.e., "systemic") countries over time for different choices of the horizontal thresholds (i.e., capital buffers) in the (left) multiplex global financial network and (right) aggregate global financial network. The solid curve is for the baseline values $b_{i}=C A R_{i}$, the dot-dashed curves are for values that are below the baseline, and the dashed curves are for values that are above the baseline. The color of a curve indicates a specific choice of the thresholds

the number of systemically important countries in our simulations on the multiplex network (dashed curves) is more than double the number that we obtain in our simulations for each type of monolayer network (dotted curves). Furthermore, for vertical thresholds of $0.33,0.50$, and 0.66 , the multiplex network has a larger number of systemically important countries than the monolayer networks. For a vertical threshold of 0.80 , the number of systemically important countries in the multiplex network is similar to those in the monolayer networks (including sometimes matching the numbers in the banking and equity networks). We also observe that the number of systemically important countries tends to increase over time both in the multiplex network and (to a lesser extent) in the equity network. It is not clear if there is a concretely identifiable trend in the other types of monolayer networks.

Effects of capital buffers. A financial entity can reduce its risk of distress by a precautionary building of its capital buffers, which it can use at the time of financial distress.

Because our horizontal thresholds encode capital buffers, we examine how capital buffers can reduce global systemic risk by considering different sizes of these thresholds. We consider seven scenarios. In scenarios one to three, the horizontal thresholds of each country $i$ are $0.67 \approx 2 / 3,0.80$, and 0.90 times $\mathrm{CAR}_{i}$ (i.e., 33\%, 20\%, and $10 \%$ below $\mathrm{CAR}_{i}$ ), respectively. In the fourth scenario, the horizontal threshold of each country $i$ is equal to $\mathrm{CAR}_{i}$. In scenarios five to seven, the horizontal thresholds of each country $i$ are 1.10 , 1.20, and 1.33 times $\mathrm{CAR}_{i}$ (i.e., 10\%, 20\%, and 33\% above $\mathrm{CAR}_{i}$ ), respectively. We count the number of systemically important countries in each scenario and for each year (see Fig. 6). We find that the horizontal threshold strongly influences the number of systemically important countries in both the multiplex and the monolayer networks. For example, as we show in Fig. 6, decreasing the horizontal thresholds (i.e., the capital buffers) by approximately one third can double the number of systemically important countries for both the aggregate and the multiplex networks. We also observe a decrease in the number of systemically important countries in the year 2016.

We observe which countries are systemically important in each of the seven scenarios (see Fig. 7). To illustrate our results, we examine countries that are systemically important in four or more years for the baseline horizontal threshold $b_{i}=\mathrm{CAR}_{i}$ (i.e., the 


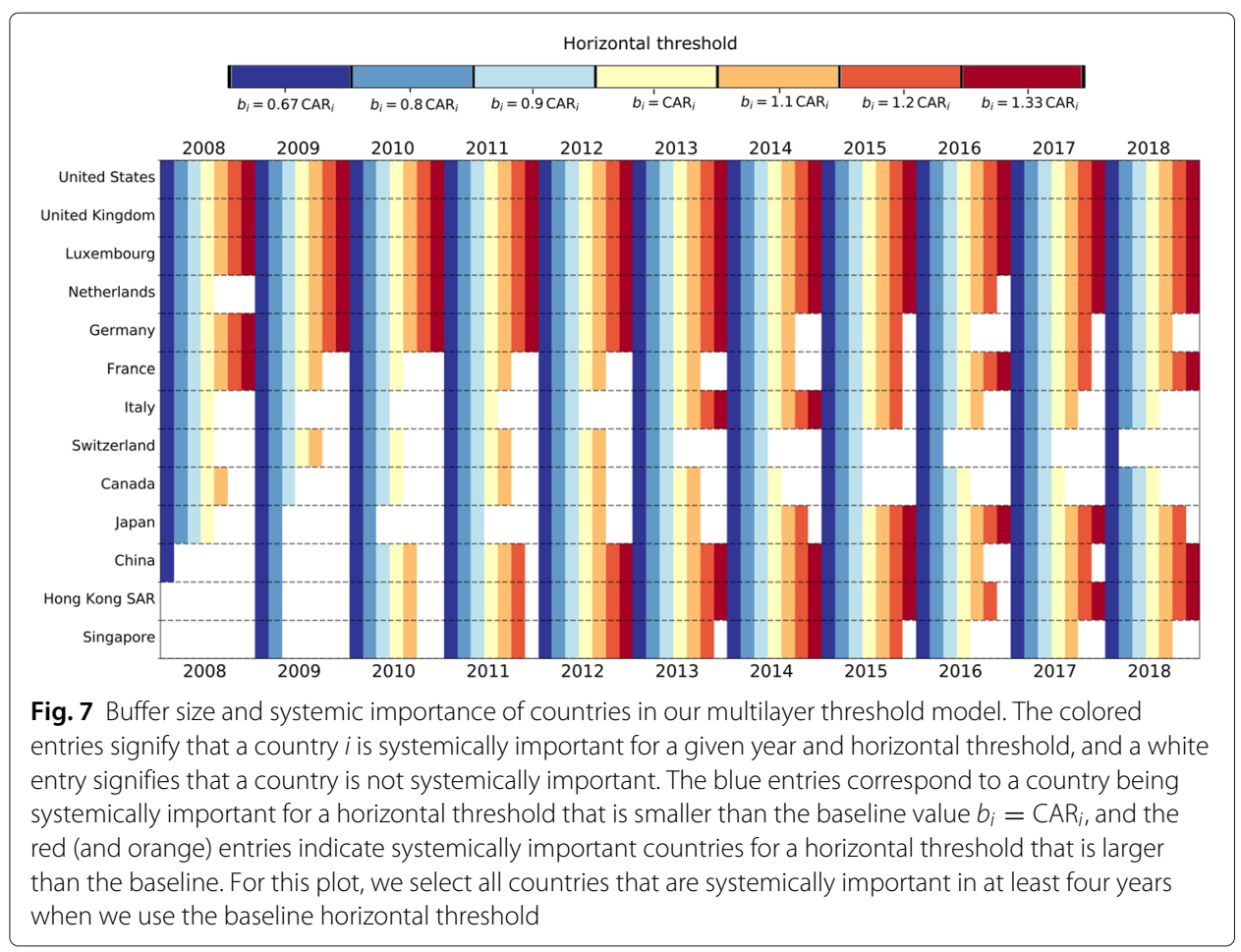

fourth scenario, which is our baseline scenario) and check whether they are systemically important in the other scenarios. In Fig. 7, we illustrate that some countries are not systemically important in the baseline scenario, but become systemically important in scenarios in which the horizontal thresholds are below the baseline. We also observe that some countries are systemically important when the horizontal thresholds equal the CARs, but they are not systematic in scenarios in which the horizontal thresholds are above the baseline.

For the year 2018, an increase of the CARs by $10 \%$ reduces the number of systemically important countries from 15 to 12 , and a $20 \%$ increase further decreases the number of systemically important countries to 8 . However, there are several countries that remain systemically important for most years even when we consider CARs that are $33 \%$ larger than the baseline values. These countries are the United States, the United Kingdom, Luxembourg, and the Netherlands. We also find that China, Hong Kong SAR, and Singapore are systemically important in more scenarios in more recent years.

Figure 7 is helpful for comparing our insights from our MultiRank and threshold-model analyses. Both MultiRank and our threshold model suggest that (i) the United States, the United Kingdom, Luxembourg, and the Netherlands are the most systemically important countries and that (ii) several Asian countries have increased in systemic importance over the years. However, although China and Hong Kong SAR are systemically important in more scenarios than Germany from 2014 to 2018, Germany's MultiRank is larger than the MultiRanks of both China and Hong Kong SAR. Similarly, Italy has a larger MultiRank than China and Singapore from 2008 to 2016, and it has a larger MultiRank than Hong Kong SAR from 2008 to 2013. However, China, Singapore, and Hong Kong SAR are systemically important in as many or more scenarios than Italy from 2010 to 2018 (with the exception of Singapore in 2013 and 2016). 


\section{Discussion}

Our modeling and computations give new insights into how different mechanisms of the spreading of financial distress can affect global financial stability. Specifically, using a multilayer threshold model that incorporates both "horizontal" (between different countries) and "vertical" (between different assets for a given country) channels of contagion, we show that a financial contagion can be more extreme for multiplex network structures than for corresponding monolayer networks. In other words, we obtain a larger number of systemically important countries when we use the multilayer threshold model. We also illustrated that several Asian countries increase in their centrality rank over time in the global financial networks, whereas several European countries decrease in their centrality rank over time. We also observed that increasing the horizontal thresholds (which we interpreted as capital buffers) decreases the number of systemically important countries.

We obtained a few similar insights in our MultiRank and threshold-model analyses. Both approaches identified the same top-four systemically important countries and pointed to a rise in systemic importance of some Asian countries. However, we also found that having a larger MultiRank value does not imply that a country is systemically important in all scenarios. When comparing our results for multiplex and monolayer networks, we found that MultiRank and PageRank correlate strongly with each other. By contrast, we observed important differences between the results of the multiplex and monolayer threshold models. Our threshold models suggest that the multiplex structure of the global financial network has a larger number of systemically important countries than its aggregate-network counterpart. These results imply that it will be useful to develop multilayer centrality measures that are based on contagion dynamics to obtain insights into systemic risk.

It is common to use threshold models to model systemic risk (Caccioli et al. 2018). In our multiplex threshold model, we observed for all years (2008-2018) that we examined that there are many more systemically important countries than in the aggregate threshold model. Therefore, our results stress the need for further research on multilayer networks and financial contagions to better understand systemic risk. For example, it would be beneficial to conduct a detailed study of how initial conditions and parameter values affect qualitative results and to compare the observations of such a study with previous financial crises. It is also essential to develop models that go beyond threshold models, as we discuss below and has been suggested by others (Petrone and Latora 2018).

In the present work, we incorporated the fact that there are multiple types of assets by examining multiplex financial networks. In future work, it will be useful to allow the horizontal and vertical thresholds to be heterogeneous across asset types in a way that incorporates the different characteristics of different assets. For instance, one can relax the assumption of a country-wide CAR, a measure that is used traditionally for banks, and incorporate heterogeneous buffers (such as the value of corporate cash holdings). One can also take into account additional considerations, such as the level of a country's reserves or its access to emergency borrowing facilities, which may provide protection against losses from financial distress.

One can also incorporate previous approaches to modeling financial distress propagation within a country (Gray et al. 2013) by considering coupling between the public ${ }^{9}$,

\footnotetext{
$\overline{9}$ The public sector is the part of the economy that consists of public services and public enterprises.
} 
private non-bank, and banking sectors. These three sectors have different types of asset exposures, so they may in turn have different risks of financial distress. Financial distress can originate in any of these sectors, and a model can examine how financial distress can propagate between these sectors within a country and across countries. For instance, a crisis that originates in the banking sector of a country can weaken the private nonbanking sector and the public sector, which in turn may exacerbate the banking distress itself (Farhi and Tirole 2018). An analogous situation can occur if a crisis originates in the public sector.

\section{Conclusions}

We studied financial contagions in multiplex networks of financial assets. In the years following the 2008 global financial crisis, some countries reduced their banking assets and increased their investments in equity. We calculated centrality measures - MultiRank for the multiplex global financial network and PageRank for the monolayer networks and found that there was a decreasing trend in the ranks of the systemic importances of some major European countries after the 2008 global financial crisis, whereas there was an increasing trend in the ranks of the systemic importances of some major Asian countries. Building on prior work (see, e.g., Caccioli et al. (2018) and Gai and Kapadia (2010)) on threshold models of financial contagions, we developed a multiplex threshold model to examine contagions in the global financial system. Our model includes both "horizontal" (i.e., intralayer) and "vertical" (i.e., interlayer) mechanisms, with associated thresholds for the propagation of financial distress. We examined a variety of parameter values, and we observed for several values of the vertical threshold that shocks in the multiplex global financial network yield a larger number of financially distressed countries than they do in its associated monolayer networks. Similarly, for several choices of the horizontal thresholds, we observed that shocks in the multiplex global financial network yield a larger number of systemically important countries than in the aggregate global financial network.

Our study highlights the importance of considering the multiplex nature of financial contagions, including by examining both "horizontal" (i.e., intralayer) and "vertical" (i.e., interlayer) spreading mechanisms, and we expect that incorporating multiple contagion channels will also be insightful for future studies of social contagions.

\section{Appendix}

MultiRank

MultiRank is a multiplex centrality measure that assigns a rank to nodes $i$ and an "influence" to layers $\alpha$. For a general multiplex network, one can compute MultiRank by solving the following equations (Rahmede et al. 2018):

$$
\begin{aligned}
z^{[\alpha]} & =\frac{1}{N}\left(W^{[\alpha]}\right)^{\phi}\left(\sum_{i=1}^{n} B_{\alpha i}^{\mathrm{in}}\left(x_{i}\right)^{\delta \gamma}\right)^{\delta}, \\
x_{i} & =\kappa \sum_{j=1}^{n} \frac{G_{j i}}{k_{j}} x_{j}+\chi v_{i},
\end{aligned}
$$


with

$$
\begin{aligned}
& k_{j}=\max \left\{1, \sum_{i} G_{j i}\right\}, \\
& v_{i}=\theta\left(\sum_{j}\left(G_{i j}+G_{j i}\right)\right), \\
& \chi=\frac{1}{\sum_{i} v_{i}} \sum_{j}\left(\left[1-\kappa \theta\left(\sum_{i} G_{j i}\right)\right] x_{j}\right),
\end{aligned}
$$

where $\theta$ is the Heaviside function.

When all nodes have an out-degree of at least 1 in at least one layer, we can reduce the above system of equations to Eq. (4) of the main manuscript. With this condition on the out-degree, it follows that

$$
\begin{aligned}
& k_{j}=\sum_{j} G_{j i}, \\
& v_{j}=1,
\end{aligned}
$$

and

$$
\chi=\frac{1}{n} \sum_{j}(1-\kappa) x_{j} .
$$

From $\sum_{j} x_{j}=1$, we obtain

$$
\begin{aligned}
z^{[\alpha]} & =\frac{1}{N}\left(W^{[\alpha]}\right)^{\phi}\left(\sum_{i=1}^{n} B_{\alpha i}^{\mathrm{in}}\left(x_{i}\right)^{\delta \gamma}\right)^{\delta}, \\
x_{i} & =\kappa \sum_{j=1}^{n} \frac{G_{j i}}{k_{j}} x_{j}+\frac{1-\kappa}{n},
\end{aligned}
$$

which is Eq. (4) of the main manuscript.

\section{DebtRank}

Although we do not compute DebtRank in the present paper, it useful to define it to help explain a key issue with it that we highlighted in the main manuscript. DebtRank, a centrality measure whose name is motivated by PageRank (Gleich 2015), is an algorithm for monolayer networks that estimates the economic loss that a node (or a set of nodes) would cause if it becomes financially distressed (or they become distressed, in the case of multiple nodes) (Bardoscia et al. 2015).

DebtRank supposes that a network's adjacency-matrix elements $A_{i j}$ encode the monetary value that an institution $i$ has invested in institution $j$ for all institutions $i$ and $j$, and it incorporates a capital-buffer vector $\mathbf{E}$ whose entries encode the capital buffers of the institutions.

Given an adjacency matrix $A$ and a capital-buffer vector E, one defines a relativeeconomic-value vector $\mathbf{v}$ with components

$$
v_{j}=\frac{\sum_{j} A_{i j}}{\sum_{l, j} A_{l j}}
$$

and an impact matrix $M$ with components

$$
M_{i j}=\min \left\{1, \psi \frac{A_{i j}}{E_{j}}\right\},
$$


where $\psi \in[0,1]$ is a scaling factor. The impact matrix, as its name suggests, determines the impact that node $j$ has on node $i$ if the former becomes distressed. The functional form of Eq. (18) incorporates the assumption that the maximum impact is 1 and that the impact increases linearly with the assets that $i$ has invested in $j$ (until one reaches the maximum impact of 1 ).

DebtRank follows an iterative procedure to compute two state variables, which we denote by $h_{i}(t)$ and $r_{i}(t)$. The variable $h_{i}(t) \in[0,1]$ indicates the financial distress of node $i$ at time $t$, and the variable $r_{i}(t) \in\{U, D, I\}$ indicates whether node $i$ is financially undistressed, distressed, or inactive.

To compute the DebtRank of the nodes in a set $\mathcal{U} \subseteq \mathcal{V}$, one initializes the state variables by letting $h_{i}(0)=0$ for all $i \notin \mathcal{U}$ and $h_{i}(0)=1$ for all $i \in \mathcal{U}$. Similarly, one initializes $r_{i}(0)=\mathcal{U}$ for all $i \notin \mathcal{U}$ and $r_{i}(0)=D$ for all $i \in \mathcal{U}$. To determine a node's state at a discrete time $t \geq 1$, one then performs the following iteration:

$$
h_{i}(t)=\min \left\{1, h_{i}(t-1)+\sum_{j \in D_{f}(t-1)} M_{i j}\right\},
$$

where $D_{f}(t-1)$ is the set of distressed nodes at the previous time (i.e., nodes with $r_{i}(t-$ $1)=D$ ). One then updates the state variable $r_{i}(t)$ using the following rule:

$$
r_{i}(t)= \begin{cases}D, & \text { if } h_{i}(t)>0 \text { and } r_{i}(t-1) \neq I \\ I, & \text { if } r_{i}(t-1)=D \\ r_{i}(t-1), & \text { otherwise }\end{cases}
$$

One iterates the dynamics until some steady-state time $\tau$ at which all nodes are either undistressed or inactive. The DebtRank of the nodes in the set $\mathcal{U} \in \mathcal{V}$ is

$$
\operatorname{DR}(\mathcal{U})=\sum_{j} h_{j}(\tau) v_{j}-\sum_{j} h_{j}(0) v_{j}
$$

The issue with DebtRank is that it is not additive, in the sense that the DebtRank of the set $\{i, j\}$ of two nodes $(i$ and $j$ ) can be smaller than the DebtRank of the individual nodes $i$ or $j$. The DebtRank algorithm assumes that a node, once distressed, becomes inactive (which implies that it does not lose more assets and that it has no further effect on other nodes) in the next time step.

This implies that once a node becomes inactive due to the distress of a particular neighbor, it becomes immune to any financial distress that could have arisen from a different neighboring node later becoming distressed. For example, even if all neighbors of node $i$ are inactive by some time $\tau_{0}$ (because they were distressed in some previous time step),

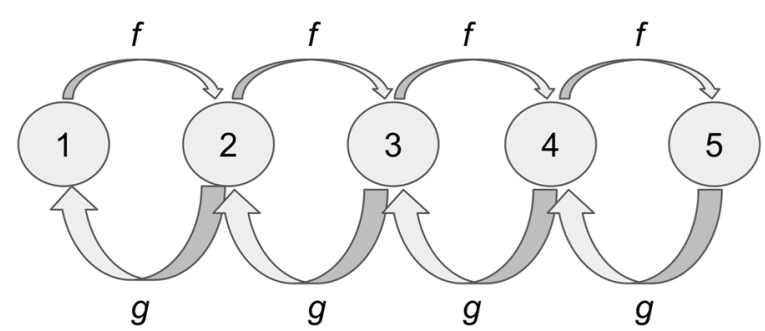

Fig. 8 Example network for illustrating DebtRank. This network has five nodes and is both weighted and directed. The quantities $f$ and $g$ denote the weights of the edges. We use arrow widths to highlight our assumption that $g>f$ 


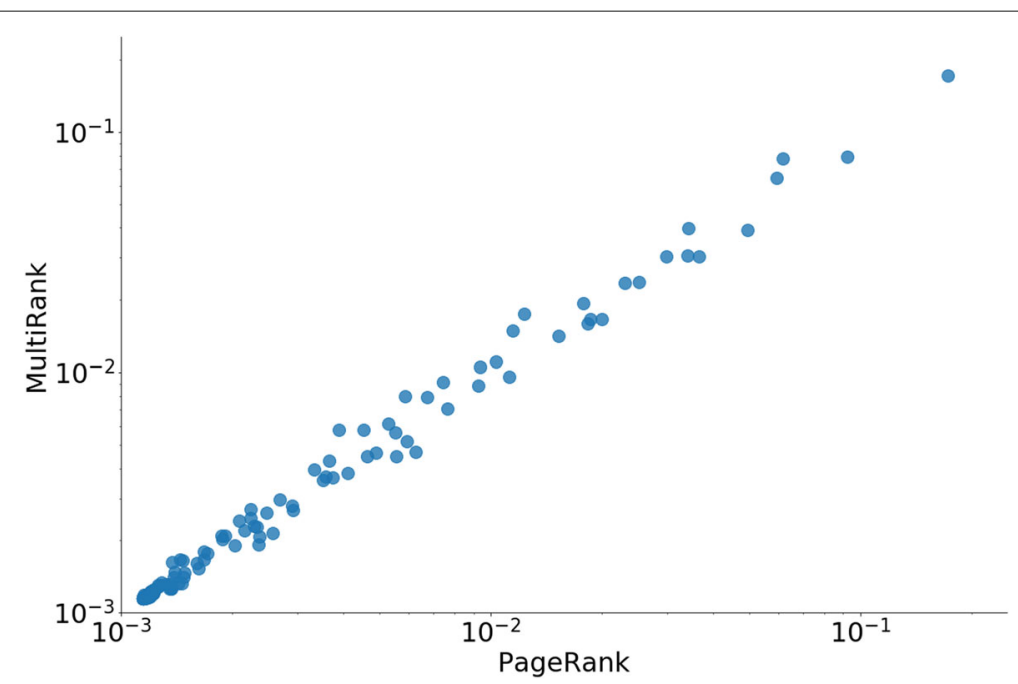

Fig. 9 Scatter plot of MultiRank scores versus PageRank scores for the global financial network in 2018. As we mentioned in the main manuscript, the MultiRank and PageRank scores are strongly correlated

the estimate of the financial losses of node $i$ may consider only a subset of node $i$ 's neighbors. This feature makes it possible for the function DR with one initially distressed node to be larger than DR with two or more initially distressed nodes.

In particular, it is possible that $\mathrm{DR}(\{i\})>\operatorname{DR}(\{i, j\})$. For example, consider the weighted and directed network in Fig. 8, where $f$ and $g$ are the weights of the edges. With $f=1$ and $g=5$, because nodes become inactive one time step after becoming distressed, we obtain

$$
\operatorname{DR}(\{1\}) \approx 0.72>\operatorname{DR}(\{1,5\}) \approx 0.61 .
$$

\section{Correlation between MultiRank and PageRank for the Global Financial Network}

As we discussed in the main manuscript, the Kendall rank correlation between the MultiRank and PageRank scores is large (specifically, above 0.90) throughout the years in our sample. In Fig. 9, we show a scatter plot of the MultiRank and PageRank scores for the global financial network for 2018, and we see that they are indeed strongly correlated with each other. In Table 1, we show the Kendall rank correlation coefficient between the

Table 1 MultiRank versus PageRank for the global financial network for each year

\begin{tabular}{lll}
\hline Year & Kendall coefficient & $p$-value \\
\hline 2008 & 0.97 & $1.66 \times 10^{-61}$ \\
2009 & 0.96 & $3.52 \times 10^{-60}$ \\
2010 & 0.96 & $4.32 \times 10^{-60}$ \\
2011 & 0.96 & $2.00 \times 10^{-60}$ \\
2012 & 0.95 & $9.69 \times 10^{-59}$ \\
2013 & 0.94 & $1.70 \times 10^{-58}$ \\
2014 & 0.94 & $7.71 \times 10^{-58}$ \\
2015 & 0.93 & $1.19 \times 10^{-56}$ \\
2016 & 0.94 & $1.44 \times 10^{-57}$ \\
2017 & 0.93 & $3.21 \times 10^{-56}$ \\
2018 & 0.93 & $2.27 \times 10^{-56}$ \\
\hline
\end{tabular}

We show the values of the Kendall rank correlation coefficient and the $p$-value between MultiRank and PageRank for each year from 2008 through 2018 
MultiRank and PageRank scores for each year. We also show the corresponding p-value of the correlations. As a robustness check, we also compute the correlations after we exclude the top-four countries (the United States, the United Kingdom, Luxembourg, and the Netherlands). We find that the correlations are the same as when we do not exclude these countries when we round to two digits after the decimal point.

\section{Abbreviations}

IMF: International Monetary Fund; CAR: Capital adequacy ratio; CDIS: Coordinated Direct Investment Survey; CPIS: Coordinated Portfolio Investment Survey; BIS: Bank for International Settlements; FSI: Financial Soundness Indicators; USD: United States dollar; WTM: Watts threshold model; BCBS: Basel Committee on Banking Supervision

\section{Acknowledgments}

We thank J. Doyne Farmer, Vikram Haksar, Anton Pichler, and Fabian Valencia for their valuable insights. The views expressed in this manuscript are those of the authors and do not necessarily represent the views of the IMF, its Executive Board, or IMF management.

\section{Authors' contributions}

All authors designed the research, analyzed the results, and wrote the paper. RMdR-C implemented the simulations and algorithms. The author(s) read and approved the final manuscript.

\section{Funding}

RMdR-C acknowledges funding from Mexico's Consejo Nacional de Ciencia y Tecnologia and Secretaria de Energia (CONACYT-SENER), Baillie Gifford, and the 2019 IMF Fund Internship Program.

\section{Availability of data and materials}

The CDIS, CPIS, and FSI data sets are publicly available. The CDIS data are available at (International Monetary Fund 2018a), the CPIS data are available at (International Monetary Fund 2018b), and the FSI data are available at (International Monetary Fund 2018c). We obtained the BIS data under confidentiality agreements with the BIS. We have made our code and the edge lists of the equity and debt layers available at https://zenodo.org/record/3963723.

\section{Competing interests}

The authors declare that they have no competing interests.

\section{Author details}

${ }^{1}$ Institute for New Economic Thinking at the Oxford Martin School, University of Oxford, Oxford, UK. ${ }^{2}$ Mathematical Institute, University of Oxford, Oxford, UK. ${ }^{3}$ International Monetary Fund, Washington D.C., USA. ${ }^{4}$ Department of Mathematics, University of California Los Angeles, Los Angeles, CA, USA.

Received: 17 March 2020 Accepted: 4 August 2020

Published online: 14 October 2020

\section{References}

Aleta A, Moreno Y (2019) Multilayer networks in a nutshell. Ann Rev Cond Matter Phys 10:45-62

Anand K, Gai P, Kapadia S, Brennan S, Willison M (2013) A network model of financial system resilience. J Econ Behav Organ 85:219-235

Aoki K, Benigno G, Kiyotaki N, et al (2010) Adjusting to capital account liberalization. CEPR Discussion Paper No. DP8087. Available at SSRN: https://ssrn.com/abstract=1714859

Bank for International Settlements (2018) International banking statistics. Available at https://www.bis.org/statistics/ rppb1807.htm. Accessed July 2019

Bardoscia M, Battiston S, Caccioli F, Caldarelli G (2015) DebtRank: A microscopic foundation for shock propagation. PloS ONE 10(6):e0130406

Bardoscia M, Caccioli F, Perotti Jl, Vivaldo G, Caldarelli G (2016) Distress propagation in complex networks: The case of non-linear DebtRank. PloS ONE 11(10):e0163825

Bargigli L, Di lasio G, Infante L, Lillo F, Pierobon F (2015) The multiplex structure of interbank networks. Quant Finance 15(4):673-691

Basel Committee on Banking Supervision (2019) Minimum capital requirements for market risk. Available at https://www. bis.org/bcbs/publ/d457.htm. Accessed July 2019

Battiston S, Farmer JD, Flache A, Garlaschelli D, Haldane AG, Heesterbeek H, Hommes C, Jaeger C, May R, Scheffer M (2016) Complexity theory and financial regulation. Science 351(6275):818-819

Battiston S, Gatti DD, Gallegati M, Greenwald B, Stiglitz JE (2012) Default cascades: When does risk diversification increase stability. J Financ Stab 8(3):138-149

Battiston S, Puliga M, Kaushik R, Tasca P, Caldarelli G (2012) DebtRank: Too central to fail? Financial networks, the FED and systemic risk. Sci Rep 2:541

Becker C, Noone C, et al. (2009) Volatility in international capital movements. Tech. Rep. Reserv Bank Aust. Available at https://www.rba.gov.au/publications/rdp/2009/pdf/rdp2009-09.pdf

Bianconi G (2018) Multilayer Networks: Structure and Function. Oxford University Press, Oxford, UK

Bonaccorsi G, Riccaboni M, Fagiolo G, Santoni G (2019) Country centrality in the international multiplex network. Appl Netw Sci 4(1):126 
Bravo C, Óskarsdóttir M. (2020) Evolution of credit risk using a personalized PageRank algorithm for multilayer networks KDD '20: Proceedings of the 26th ACM SIGKDD International Conference on Knowledge Discovery \& Data Mining, Workshop on Machine Learning in Finance (in press). arXiv:2005.12418

Brummitt CD, Kobayashi T (2015) Cascades in multiplex financial networks with debts of different seniority. Phys Rev E 91(6):062813

Brummitt CD, Lee KM, Goh KI (2012) Multiplexity-facilitated cascades in networks. Phys Rev E 85(4):045102

Caccioli F, Barucca P, Kobayashi T (2018) Network models of financial systemic risk: A review. J Comput Soc Sci 1(1):81-114

Caccioli F, Shrestha M, Moore C, Farmer JD (2014) Stability analysis of financial contagion due to overlapping portfolios. J Bank Finance 46:233-245

Claessens S, Dooley MP, Warner A (1995) Portfolio capital flows: Hot or cold? World Bank Econ Rev 9(1):153-174

De Haas R, Van Horen N (2012) International shock transmission after the Lehman Brothers collapse: Evidence from syndicated lending. Am Econ Rev 102(3):231-37

Diem C, Pichler A, Thurner S (2020) What is the minimal systemic risk in financial exposure networks? J Econ Dyn Control 116:103900

De Domenico M, Granell C, Porter MA, Arenas A (2016) The physics of spreading processes in multilayer networks. Nat Phys 12:901-906

Eisenberg L, Noe TH (2019) Systemic risk in financial systems. Manag Sci 47(2):236-249

Farhi E, Tirole J (2018) Deadly embrace: Sovereign and financial balance sheets doom loops. Rev Econ Stud 85(3):1781-1823

Farmer JD, Kleinnijenhuis AM, Nahai-Williamson P, Wetzer T (2020) Foundations of system-wide financial stress testing with heterogeneous institutions. Tech. Rep. Bank of England. Available at https://www.bankofengland.co.uk/-/ media/boe/files/working-paper/2020/foundations-of-system-wide-financial-stress-testing-with-heterogeneousinstitutions.pdf

Feinstein Z, Sojmark A (2019) A dynamic default contagion model: From Eisenberg-Noe to the mean field. arXiv: 1912.08695

Fernando CS, May AD, Megginson WL (2012) The value of investment banking relationships: Evidence from the collapse of Lehman Brothers. J Finance 67(1):235-270

Forbes K (2014) Financial 'deglobalization'? Capital flows, banks and the Beatles. Speech to Queen Mary University of London. Available at https://www.bankofengland.co.uk/speech/2014/financial-deglobalization-capital-flows-banksand-the-beatles

Forbes K, Reinhardt D, Wieladek T (2017) The spillovers, interactions, and (un) intended consequences of monetary and regulatory policies. J Monet Econ 85:1-22

Gai P, Kapadia S (2010) Contagion in financial networks. Proc R Soc London A Math Phys Eng Sci 466:2401-2423

Gleeson JP (2013) Binary-state dynamics on complex networks: Pair approximation and beyond. Phys Rev X 3(2):021004

Gleich DF (2015) PageRank beyond the Web. SIAM Rev 57(3):321-363

Gray D, Gross M, Paredes J, Sydow M (2013) Modeling banking, sovereign, and macro risk in a CCA global VAR. Tech. Rep. WP/13/218, International Monetary Fund. Available at https://www.imf.org/external/pubs/ft/wp/2013/wp13218.pdf

Greenwood R, Landier A, Thesmar D (2015) Vulnerable banks. J Finan Econ 115(3):471-485

Haldane AG, May RM (2011) Systemic risk in banking ecosystems. Nature 469(7330):351-355

International Monetary Fund (2018a) Coordinated Direct Investment Survey. Available at https://data.imf.org/?sk= 40313609-F037-48C1-84B1-E1F1CE54D6D5. Accessed July 2019

International Monetary Fund (2018b) Coordinated Portfolio Investment Survey. Available at https://data.imf.org/?sk= B981B4E3-4E58-467E-9B90-9DE0C3367363. Accessed July 2019

International Monetary Fund (2018c) Financial soundness indicators. Available at https://data.imf.org/?sk=51B096FA2CD2-40C2-8D09-0699CC1764DA. Accessed July 2019

Kaushik R, Battiston S (2013) Credit default swaps drawup networks: Too interconnected to be stable? PLoS ONE 8(7):e61815

Kivelä M (2014) PYMNET: Multilayer networks library for Python. Available at http://www.mkivela.com/pymnet. Accessed Dec 2019

Kivelä M, Arenas A, Barthelemy M, Gleeson JP, Moreno Y, Porter MA (2014) Multilayer networks. J Complex Netw 2(3):203-271

Korniyenko Y, Patnam M, del Rio-Chanona RM, Porter MA (2018) Evolution of the global financial network and contagion: A new approach. Tech. Rep. WP/18/113, International Monetary Fund. Available at https://www.imf.org/ /media/ Files/Publications/WP/2018/wp18113.ashx

Lane PR, Milesi-Ferretti GM (2017) International financial integration in the aftermath of the global financial crisis. Tech. Rep. WP/17/115, International Monetary Fund. Available at https://www.imf.org/en/Publications/WP/Issues/2017/05/ 10/International-Financial-Integration-in-the-Aftermath-of-the-Global-Financial-Crisis-44906

Lee K-M, Brummitt CD, Goh K-I (2014) Threshold cascades with response heterogeneity in multiplex networks. Phys Rev E 90(6):062816

Leon C, Berndsen R, Renneboog L (2014) Financial stability and interacting networks of financial institutions and market infrastructures. Borradores de Economia 848. Report available at https://econpapers.repec.org/paper/bdrborrec/848. htm

Li Y, Liu G, Pin P (2018) Network-based risk measurements for interbank systems. PLoS ONE 13(7):e0200209

Lund S, Daruvala T, Dobbs R, Härle P, Kwek JH, Falcón R (2013) Financial globalization: Retreat or reset. McKinsey Global Institute. Available at https://www.mckinsey.com/featured-insights/employment-and-growth/financial-globalization

Mallaby S (2016) Globalization resets. Finance Dev 53(4):6-10. http://www.contexto.org/pdfs/2016/ IMFgloblwinersloosers.pdf

Markose SM (2012) Systemic risk from global financial derivatives: A network analysis of contagion and its mitigation with super-spreader tax. Tech. Rep. WP/12/282, International Monetary Fund. Available at https://www.elibrary.imf.org/ view/IMF001/20120-9781475577501/20120-9781475577501/20120-9781475577501_A001.xml?

Newman MEJ (2018) Networks. second edn. Oxford University Press, Oxford, UK 
Petrone D, Latora V (2018) A dynamic approach merging network theory and credit risk techniques to assess systemic risk in financial networks. Sci Rep 8:5561

Pichler A, Poledna S, Thurner S (2018) Systemic-risk-efficient asset allocation: Minimization of systemic risk as a network optimization problem. arXiv:1801.10515

Poledna S, Hinteregger A, Thurner S (2018) Identifying systemically important companies in the entire liability network of a small open economy. Entropy 20(10):792

Poledna S, Molina-Borboa JL, Martínez-Jaramillo S, Van Der Leij M, Thurner S (2015) The multi-layer network nature of systemic risk and its implications for the costs of financial crises. J Finan Stab 20:70-81

Porter MA (2018) WHAT IS... a multilayer network? Not AMS 65(11):1419-1423

Porter MA, Gleeson JP (2016) Dynamical systems on networks: A tutorial. Frontiers in Applied Dynamical Systems: Reviews and Tutorials, vol. 4. Springer International Publishing, Cham, Switzerland

Rahmede C, lacovacci J, Arenas A, Bianconi G (2018) Centralities of nodes and influences of layers in large multiplex networks. J Complex Netw 6(5):733-752

Silva TC, da Silva Alexandre M, Tabak BM (2018) Bank lending and systemic risk: A financial-real sector network approach with feedback. J Finan Stab 38:98-118

Tebogo B (2012) The failure and collapse of the Lehman Brothers. Available at SSRN at https://ssrn.com/abstract=2060758 van Lidth de Jeude JA (2019) Analysing Mesoscale Structures in Economic and Financial Networks. PhD thesis. Available at http://e-theses.imtlucca.it/id/eprint/266

Watts DJ (2002) A simple model of global cascades on random networks. Proc Natl Acad Sci U S A 99(9):5766-5771

Wiersema G, Kleinnijenhuis AM, Wetzer T, Farmer JD (2019) Scenario-free analysis of financial stability with interacting contagion channels. Available at SSRN at https://ssrn.com/abstract=3408533

Yağan O, Gligor V (2012) Analysis of complex contagions in random multiplex networks. Phys Rev E 86(3):036103

\section{Publisher's Note}

Springer Nature remains neutral with regard to jurisdictional claims in published maps and institutional affiliations.

\section{Submit your manuscript to a SpringerOpen ${ }^{\circ}$ journal and benefit from:}

- Convenient online submission

- Rigorous peer review

- Open access: articles freely available online

- High visibility within the field

- Retaining the copyright to your article

Submit your next manuscript at $\boldsymbol{\triangleright}$ springeropen.com 